\title{
Measuring Consumer Behavioural Intention to Accept Technology: Towards Autonomous Vehicles Technology Acceptance Model (AVTAM)
}

\author{
Patrice Seuwou $^{1,2}$, Christos Chrysoulas ${ }^{2}$, Ebad Banissi $^{2}$ and George Ubakanma ${ }^{2}$ \\ ${ }^{1}$ Northampton University, Northampton NN1 5PH, UK \\ patrice.seuwoulnorthampton.ac.uk \\ ${ }^{2}$ London South Bank University, London SE1 0AA, UK \\ \{seuwoup, chrysouc, banisse, george.ubakanma\} @lsbu.ac.uk
}

\begin{abstract}
The work presented in the paper aims at exploring information technology acceptance in the context of Autonomous Vehicles (AV) with the objectives of identifying and testing the constructs that will influence future AVs acceptance. Most models of technology acceptance focus on barriers of successful information technology implementation in organisations and technologies that have already been deployed. There is only a small number of studies conducted on emerging and disruptive technologies such as AV. We address this issue by deriving context-related determinants from an extensive literature analysis and further describing a technology acceptance modeling process to provide an explanation for drivers' and factors influencing people behavioural intention to accept AV technology. Based on our evaluation we take the determinants self-efficacy, perceived safety, trust, anxiety and legal regulation into consideration. The outcome and main contribution of this paper is the proposal of a theoretical AV technology acceptance model (AVTAM). This study concluded that the performance of these AV technologies, their safety on the road and consumer's trust for the AV manufacturers will play a very important role for mass AV adoption.
\end{abstract}

Keywords: Unified Theory of Acceptance and Use of Technology (UTAUT), UTAUT2, Autonomous Vehicles, Technology Acceptance Model, AVTAM, Context.

\section{Introduction}

For decades, the user acceptance of information technology has been a vital field of study for psychologists and sociologists investigating new insights into the acceptance of behaviour at individual and organisational levels [1]. As the application of computer technology continues to proliferate and diversify, vehicles are becoming increasingly intelligent and it is expected that soon they will be equipped with autonomous technologies allowing them to drive passengers from one location to another without any human intervention. AV also known as self-driving cars or driverless cars are vehicles equipped with several technologies including radar, laser light, GPS, odometry, 
computer vision and are capable of sensing their environment and navigating without human input. Nowadays, many car drivers do not realise that their cars are already equipped with various levels of assistance. Indeed, the current generation of vehicles can already detect, anticipate and correct autonomously other road users' maneuvers. Certainly, AV is a disruptive technology. Not all technologies predicted by popular media are immediately welcomed into society, and AV is one such technology. Despite numerous models being proposed to predict consumer use behaviour of technology, there is a gap in the literature as the latest models and theories are still not able to fully capture the complexity of the factors influencing people behavioural intention to adopt AV $[1,2,7]$. The Unified Theory of Acceptance and Use of Technology (UTAUT) and UTAUT2 are models that have been used for years to help us understand the drivers for acceptance of technologies by its users [4]. It appears that AV adoption cannot be measured only using existing models. Furthermore, there are several important factors also not represented on the latest car technology acceptance research model, therefore, the absolute need to develop a model specifically for $\mathrm{AV}$ is required. The paper proceeds as follows: First, technology acceptance concept is thoroughly reviewed to provide insights in the development process and the predictive character of acceptance models, second, determinants that effect acceptance of AV are examined. We perform a literature review as well as an analysis of qualitative results from a technology acceptance evaluation to derive and define the constructs "self-efficacy", "perceived safety", "trust", "anxiety" and "legal regulation". They form the basis for a theoretical AV Technology Acceptance Model (AVTAM) which is presented based on the UTAUT2 structure. Finally, the implications followed by future work are discussed. The rest of the paper is organised as follows: Section 2 gives an overview of the literature Review on AV acceptance, Section 3 discusses the main constructs identified for the proposed AVTAM model with the hypothesis, Section 4 provides the methods and techniques used to test the proposed research model, the results are presented in section 5 followed by a brief conclusion with future work.

\section{$2 \quad$ Literature review}

Automation of vehicles and driverless technology has attracted a lot of people from the stakeholders to the media. Stakeholders have been yearning to develop and take the automated driving technology a notch higher and meet the increasing demands of consumers. Public exposure to the different forms of electric vehicles has slowly gained momentum in recent years with various manufacturers carrying out test runs and pilot programs in Europe. Such programs help towards examining the user acceptance of these modern vehicles and act as prerequisites to ensure such investment pay off. Many authors believe that customer acceptance is likely to be the biggest obstacle to autonomous vehicle penetration $[1,2,3]$. At first, many consumers may be reluctant to put their lives in the hands of a robot. Recent studies and surveys have shown a split in opinion as to whether people would like autonomous capability to be available in their vehicles or not. User acceptance is a key ingredient to the successful adoption of autonomous vehicles. In the last three decades, the global car market has gone through some rapid changes especially in driver experiences, thanks to the technological 
advancement. For instance, a typical middle-class classic car has standard features such as automatic gearbox, an electronic stability program and power windows [4].

For the high-end vehicles, they can have intelligent features such as an automatic startup framework, self-parking technology and active lane assistance. The innovations have led to comfortable driving. However, the automobiles still need human interaction to foster their movement. Today's innovations focus on automobiles that are fully autonomous and free from direct human interaction. Driverless vehicles are those that operate without direct input by the driver to control the acceleration, steering or braking and are designed in such a way that the controller does not continuously observe the roadway when the car is moving in self-driving mode. The driver-less vehicle technology has been too advanced; opening new opportunities for the automotive dealers, individual users and also the potential manufacturers [5].

There are diverse factors that influence vehicle-purchasing behaviour amongst the people. There are the situational factors that include regulatory environments. Besides the situational factors, psychological factors also play an essential role in the purchase of autonomous vehicles and include the personal attitudes. The extensive adoption of electric cars can be understood through the examination of the different factors that influence consumer acceptance. The acceptance is critical towards the commercial success or failure of the AVs.

According to [4], there are different factors that influence and motivate the environmentally conscious behaviours. The elements encompass the personal values; environmental concerns as well as the belief that one can make a difference in the use of such vehicles. Also, these factors contribute towards an individual's purchase intentions for the alternative fuel vehicles. Gas prices significantly impact the individual's interest in buying hybrid electric cars. There are other customer preferences such as reduced air pollution, reduced maintenance and better handling. There is no doubt that environmentalists and people that have strong preferences for energy security will automatically adopt the hybrid electric cars. In their research, [6] concluded that among the top attributes considered before a new vehicle purchase include price, reliability and the fuel economy.

Different societies have distinct attributes relating to their preferences for the autonomous vehicles. According to the unified theory of acceptance, the degree of technology acceptance varies. People in Hong Kong prioritize the environmental benefits of these innovations followed by the economic and social benefits. Negative factors have also been identified as barriers towards the purchase of the electric vehicles. These include lack of AV infrastructure, limited selection of the vehicles and potential increases in the electrical rates. Results of the study by [7] highlighted four determinant factors affecting purchasing behaviours of the potential buyers: high costs, charge inconvenience, psychological factors and short battery range. A study in China also revealed some deterministic factors or concerns when purchasing autonomous vehicles such as the cost of operations, overall buying cost, possible speeds and battery capacity. In the USA, the potential buyers considered the charge time and range as concerns other than the value of the hybrid vehicles. Findings by [8] also show that the attributes, personal norms, the perceived behavioural control as well as the planning abilities influenced the decision to buy an autonomous vehicle. Customers will always be concerned about 
the potential advantages as well as the promises such as safety benefits, times savings, fuel savings.

\section{Formulation of the Autonomous Vehicles Technology Acceptance Model (AVTAM)}

Our study was conducted in the UK and our target population was potential AV users which represented any car users. Several interviews were conducted with academics and practitioner from the fields of Psychology, Sociology and Computer Science. Numerous constructs were proposed. The following research model was created (see Figure 2)

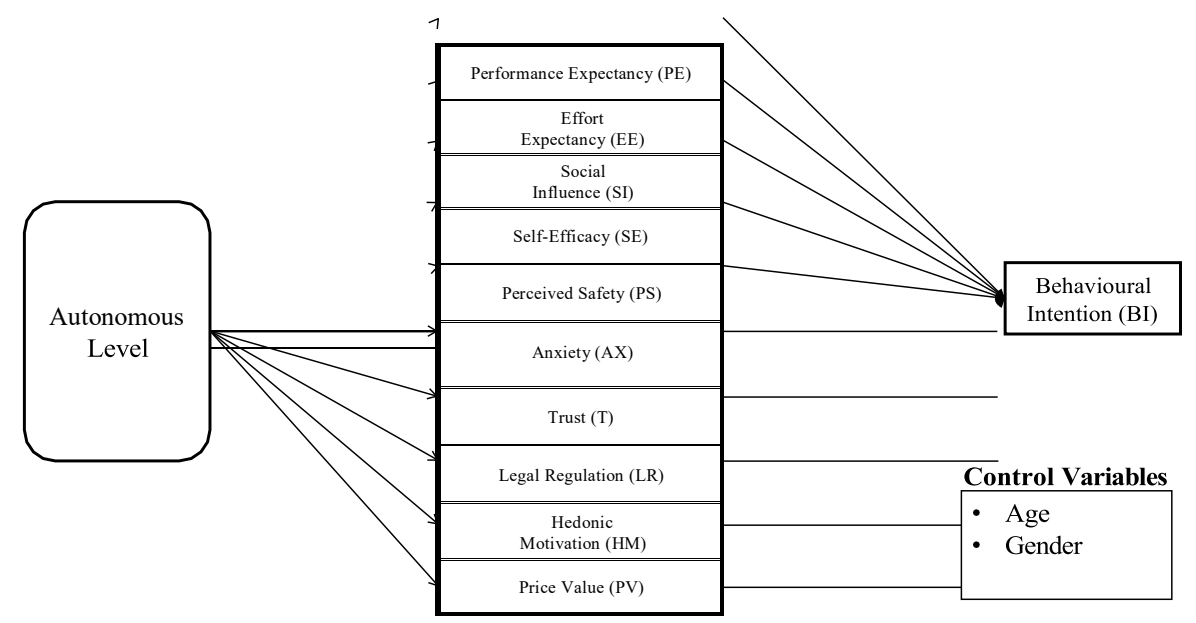

Fig. 2. Research model for Measuring consumers' behavioural intention to adopt Autonomous Vehicles: Autonomous Vehicle Technology Acceptance Model (AVTAM)

In order to maximise understanding of different viewpoints and explore other potentials factors not previously identified by existing models, an initial survey was conducted at the beginning of the study. Our target population was mainly in England, the sample size was 408 participants from all age groups, race and cultural backgrounds with a minimum of 18 years of age. In this study, a convenience sampling technique was used data was collected from staffs and students at various universities in UK, mainly due to the challenges of having access to existing AV users and technology manufacturers. Furthermore, due to the complexity of the questionnaires more appropriate for a certain demographic. The following factors and hypotheses were identified. 


\section{Performance Expectancy (PE)}

Performance expectancy is defined as the degree to which an individual believes that using autonomous vehicles will help him or her to attain gains in daily life activities, increase productivity, decrease possibilities of accidents on the road and makes driving more secure. PE also takes into consideration security features of the car; provide some level of satisfaction about trust, and privacy protection. Gender and age is theorised to play a moderating role. The influence of performance expectancy on behavioural intention will be moderated by gender and age, such that the effect will be stronger for men and in particular for younger men.

H1: Performance Expectancy will be positively related to behavioural intention of using autonomous vehicles.

Effort Expectancy (EE)

Effort Expectancy is defined as the degree of ease associated with the use of autonomous vehicles. The ease is associated with learning how to use autonomous vehicles and how clear and understandable the interaction with the technology is. The influence of effort expectancy on behavioural intention will be moderated by gender, age and experience. It is suggested that effort expectancy is more salient for women than for men. The gender differences predicted here could be driven by cognitions related to gender roles. Increased age has been shown to be associated with difficulty in processing complex stimuli and allocating attention to information. Thus, the study proposes that effort expectancy will be most salient for women, particularly those who are older and with relatively little experience with autonomous vehicles. The influence of effort expectancy on behavioural intention will be moderated by gender, age and experience, such that the effect will be stronger for women, particularly younger women and particularly at early stages of experience.

H2: Effort Expectancy will be positively related to behavioural intention of using autonomous vehicles.

\section{Social Influence (SI)}

Social Influence is defined as the degree to which an individual perceives that important others believe he or she should use the technology. Social influence occurs when one's emotions, opinions, or behaviours are affected by others. Social influence takes many forms and can be seen in conformity, socialisation, peer pressure obedience, persuasion, sales and marketing, and review of information. Furthermore, social influence as a direct determinant of behavioural intention contains the explicit or implicit notion that the individual's behaviour is influenced by the way in which they believe others will view them as a result of having used autonomous vehicles. The impact of social influence on behavioural intention will be moderated by gender, age and experience, such that the effect will be stronger for women, particularly older women, particularly older women in mandatory stages of experience. The following elements are included in this factor: Journalists, Expert reviewers, Friends, Family, media

H3: Social Influence will be positively related to behavioural intention of using autonomous vehicles. 


\section{Self-Efficacy (SE)}

The determinant self-efficacy is defined as a person's belief in his/her ability and competence to use a technology (e.g. a radio) to accomplish a particular task. It can play a major role in how one approaches a goal or a task as it is developed from external experiences and self-perception. In the Autonomous car context, people with high selfefficacy - those who believe they can perform well - are more likely to view a difficult task as something to be mastered rather that something to be avoided. Aligning results from this construct with results from the perceived safety construct, the researcher believes that this will gives us an interesting insight into how an information systemrelated task is judged based on users' personality characteristics.

H4: Self-Efficacy will be positively related to behavioural intention of using autonomous vehicles.

\section{Perceived Safety (PS)}

Perceived safety is defined as the degree to which an individual believes that using a system will affect his or her well-being. The researcher named the construct perceived safety considering the self-reflective character of perceiving a situation hazardous. Within the autonomous car, this also comprises the judgment of one's own driving skills and safety feeling in relation to other drivers. The impact of perceived safety is assumed as critical in the process of predicting the behavioural intention to use, as the user will estimate the potential effect of safety-related consequences through using the technology on public road and dangerous environments. The following elements are included in this factor: Vehicle security and safety, cyber security concerns

H5: Perceived Safety will be positively related to behavioural intention of using autonomous vehicles.

\section{Anxiety (AX)}

Anxiety is defined in the autonomous car context as the degree to which a person responds to a situation with apprehension, uneasiness or feelings of arousal. The factors Anxiety and Behavioural Anxiety differ with regards to their origin, as Anxiety was derived from a computer anxiety construct described within the SCT and was used earlier in the UTAUT validation. Behavioural Anxiety otherwise reflects anxiety in a more general understanding towards the autonomous vehicle or system behaviour which addresses e.g. the fear to lose control of the car.

H6: Anxiety will be negatively related to behavioural intention of using autonomous vehicles.

\section{Trust (T)}

Acceptance of the truth of statements from AV manufacturers without evidence or investigation. The belief in the reliability, truth, or ability of the car. The belief that the user can trust the car and the algorithm to function as advertised while protecting consumers' privacy.

H7: Trust will be positively related to behavioural intention of using autonomous vehicles. 


\section{Legal Regulation (LR)}

The rules or directives made and maintained by an authority and transportation regulatory bodies. The following elements are included in this factor:

Legislation, policies, law, liability, regulations, effort by the government and car manufacturers to better support users

H8: Legal Regulation will be positively related to behavioural intention of using autonomous vehicles.

\section{Hedonic Motivation (HM)}

Hedonic Motivation is defined as the fun or pleasure derived from using a technology, and it has been shown to play an important role in determining technology acceptance and use. Thus, the influence of hedonic motivation on behavioural intention will be moderated by gender, age and experience.

H9: Hedonic Motivation will be positively related to behavioural intention of using autonomous vehicles.

\section{Price Value (PV)}

The cost and pricing structure may have a significant impact on consumers' technology use. In marketing research, the monetary cost/price is usually conceptualized together with the quality of products or services to determine the perceived value of products or services. We followed these ideas and define price value as consumers' cognitive tradeoff between the perceived benefits of the applications and the monetary cost for using them. The price value is positive when the benefits of using a technology are perceived to be greater than the monetary cost and such price value has a positive impact on intention. Thus, price value has been added as a predictor of behavioural intention to use autonomous vehicles. The following elements are included in this factor: Cost of the vehicle, operational cost and maintenance cost, cost of network protocols being used LTE/WAVE/Wifi

H10: Price Value will have a significant influence on behavioural intention of using autonomous vehicles.

\section{Behavioural Intention (BI)}

Behavioural intention is defined as an individual's intention to perform a given act which can predict corresponding behaviours when an individual acts voluntarily. Besides that, behavioural intention is the subjective probability of carrying out behaviour and also the cause of certain usage behaviour [9]. Thus, intentions show the motivational factors that influence behaviour and are indicators of how hard people are willing to try and the effort they put in to engage in a behaviour. Also, it was found that behavioural intention is to be the main factor of individual mobile services usage and that usage intentions are rational indicators of future system use. 


\section{$4 \quad$ Method}

In this study, we first examined the basic UTAUT model with the fundamental constructs in the original UTAUT model [10] and its extension, the UTAUT2 together with the Car Technology Acceptance Model (CTAM). To test and validate the proposed model, our target population was the current vehicles drivers and general transportation consumers. Our study was conducted in the UK. All items of the model were measured using a seven-point Likert scale, with the anchors being "strongly disagree" and "strongly agree." There were 482 valid responses for the second survey. Data analysis for the final conceptual model was performed by Structured Equation Modelling (SEM) using the R Studio programming and the Statistics Package for Social Science (SPSS) system to analyse the data using reliability analysis, correlation analysis, and regression analysis.

\section{$5 \quad$ Results}

In this section, we use Cronbach Alpha to measure the internal consistency that is, how closely related set of items are as a group. All the values are greater than 0.7 , which clearly shows the reliability of the scale/research instrument. Dillon-Goldstein rho should be higher than 0.8 , as the minimum is 0.91 this is satisfactory.

\begin{tabular}{clc} 
Table 1. Cronbach's alpha & \\
\hline$\#$ & \multicolumn{1}{c}{ Measure } & Cronbach's Alpha \\
\hline 1 & Performance Expectancy & 0.91 \\
2 & Effort Expectancy & 0.93 \\
3 & Social Influence & 0.82 \\
4 & Self-Efficacy & 0.86 \\
5 & Perceived Safety & 0.80 \\
6 & Anxiety & 0.88 \\
7 & Trust & 0.85 \\
8 & Legal Regulation & 0.90 \\
9 & Hedonic Motivation & 0.96 \\
10 & Price Value & 0.87 \\
11 & Behavioral Intention & 0.92 \\
\hline
\end{tabular}

Table 2. below presents the correlation matrix showing the correlation coefficients between different constructs part of the proposed model. The most correlated variables are PE \& BI (0.81), so with the higher performance expectancy there is higher behavioural intentions, and PE \& PS (0.73) and the least correlated variable is the Age. The anxiety is negatively correlated and that is good, the maximum is for Perceived safety $(-0.74)$, which corresponds to what could be expected, as higher Perceived Safety implies less Anxiety. 
Table 2. Correlations Matrix

\begin{tabular}{|c|c|c|c|c|c|c|c|c|c|c|c|}
\hline Measure & $\mathrm{PE}$ & $\mathrm{EE}$ & SI & SE & PS & $\mathrm{AX}$ & $\mathrm{T}$ & LR & $\mathrm{HM}$ & PV & $\mathrm{BI}$ \\
\hline $\mathrm{PE}$ & 1 & & & & & & & & & & \\
\hline $\mathrm{EE}$ & 0.66 & 1 & & & & & & & & & \\
\hline SI & 0.73 & 0.62 & 1 & & & & & & & & \\
\hline SE & 0.55 & 0.62 & 0.6 & 1 & & & & & & & \\
\hline PS & 0.68 & 0.6 & 0.48 & 0.42 & 1 & & & & & & \\
\hline $\mathrm{AX}$ & 0.51 & -0.5 & 0.38 & 0.33 & 0.74 & 1 & & & & & \\
\hline $\mathrm{T}$ & 0.64 & 0.57 & 0.55 & 0.53 & 0.6 & 0.45 & 1 & & & & \\
\hline LR & 0.55 & 0.48 & 0.54 & 0.46 & 0.43 & -0.3 & 0.72 & 1 & & & \\
\hline HM & 0.58 & 0.45 & 0.53 & 0.42 & 0.36 & -0.3 & 0.5 & 0.45 & 1 & & \\
\hline PV & 0.56 & 0.49 & 0.5 & 0.4 & 0.51 & 0.43 & 0.54 & 0.5 & 0.42 & 1 & \\
\hline BI & 0.81 & 0.63 & 0.72 & 0.55 & 0.7 & 0.57 & 0.66 & 0.55 & 0.64 & 0.66 & 1 \\
\hline
\end{tabular}

Table 3. Results of the original structural model

\begin{tabular}{lccccl}
\hline \multicolumn{1}{c}{ Construct } & $\begin{array}{l}\text { Code } \\
\text { Name }\end{array}$ & Hypotheses & $\begin{array}{c}\text { Relationship } \\
\text { (Positive) }\end{array}$ & $\begin{array}{l}\text { Standardized } \\
\text { regression } \\
\text { weights }(\beta)\end{array}$ & Supported \\
\hline $\begin{array}{l}\text { Performance Ex- } \\
\text { pectancy }\end{array}$ & PE & H1 & PE $\rightarrow$ BI & 0.256 & YES*** \\
$\begin{array}{l}\text { Effort Expectancy } \\
\text { Social Influence }\end{array}$ & EE & H 2 & EE $\rightarrow$ BI & 0.038 & YES \\
Self-Efficacy & SE & H3 & SI $\rightarrow$ BI & 0.199 & YES*** \\
$\begin{array}{l}\text { Perceived Safety } \\
\text { Anxiety }\end{array}$ & PS & H5 & SE $\rightarrow$ BI & 0.020 & YES \\
Trust & T & H6 & PS $\rightarrow$ BI $\rightarrow$ BI & 0.176 & YES** \\
Legal Regulation & LR & H8 & T $\rightarrow$ BI & 0.074 & YES** \\
Hedonic Motivation & HM & H9 & LR $\rightarrow$ BI & 0.043 & YES $\rightarrow$ BI \\
Price Value & PV & H10 & PV $\rightarrow$ BI & 0.184 & YES** \\
\hline
\end{tabular}

$* * *$ Significant at 0.001 level (two tailed), **Significant at 0.01 level (two tailed)

Both the goodness of fit indices and parameter estimates coefficients were examined to check whether the hypothesised structural model fitted the data and to test the hypotheses. The fit indices indicated that the hypothesised structural model provided the good fit to the data. All hypotheses were supported. 


\section{Conclusion and Future work}

Transportation has immense implications for social welfare, economic development, and environmental sustainability. Congestion, environmental degradation, social inequity, and public health issues are problems that sustainable transport policies urgently need to resolve. In this article, we explored AVs and their potential as a solution for smart and sustainable development, we have also explored the factors that will influence consumers' adoption. The results suggest that the proposed model of the Autonomous Vehicles acceptance demonstrates a considerable explanatory and predictive power. Thus, the integration of the internal and external factors with the UTAUT2 is both theoretically appealing as well empirically significant. Future studies could focus in exploring the effect of factors such as cultural influence and socio-economic status on technology adoption.

\section{References}

1. Becker, F. and Axhausen, K. W.: ' Literature review on surveys investigating the acceptance of autonomous vehicles'(2017).

2. Morgan Stanley: Autonomous Cars: Self-Driving the New Auto Industry Paradigm, s.1.: Morgan Stanley Blue Paper (2013).

3. RTRAC: Automated Driving Roadmap: Status 3rd Draft for public consultation (2015).

4. Fraedrich, E. and Lenz, B.: Societal and Individual Acceptance of Autonomous Driving, pp.621-640. Springer Journal on Autonomous Driving (2016).

5. 2025ad.com: 2025AD |2025AD - The Automated Driving Community. [online] Available at: https://www.2025ad.com/categories/latest/technology/ last accessed 2019/10/24.

6. Rödel, C., Stadler, S., Meschtscherjakov, A. and Tscheligi, M.: Towards autonomous cars: the effect of autonomy levels on acceptance and user experience. In Proceedings of the 6th International Conference on Automotive User Interfaces and Interactive Vehicular Applications (pp. 1-8). ACM. (2014).

7. Cho, Y., Park, J., Park, S. and Jung, E.S.: Technology Acceptance Modeling based on User Experience for Autonomous Vehicles. 36(2). Journal of the Ergonomics Society of Korea, (2017).

8. Niculescu, A.I., Dix, A. and Yeo, K.H.: Are You Ready for a Drive?: User Perspectives on Autonomous Vehicles. In Proceedings of the 2017 CHI Conference Extended Abstracts on Human Factors in Computing Systems (pp. 2810-2817). ACM (2017).

9. Yi, M. Y., Jackson, J. D., Park, J. S., \& Probst, J. C. Understanding infor-mation technology acceptance by individual professionals: Toward an inte-grative view. Information \& Management, 43, 350- 363, (2006).

10. Venkatesh, V., Morris, M. G., Davis, G. B., \& Davis, F. D. User acceptance of information technology: Toward a unified view. 27(3), 425-478. MIS Quarterly, (2003). 